\title{
loT Empowered Real Time Environment Monitoring System
}

\author{
Athena Karumbaya \\ Department of Electronics and Communication \\ Vellore Institute of Technology
}

\author{
Gowri Satheesh \\ Department of Electronics and Communication \\ Vellore Institute of Technology
}

\begin{abstract}
The Internet of Things (IoT) is a computing concept that describes a future where every day physical objects will be connected to the Internet and be able to identify themselves to other devices. In the future, every device is more likely to be connected to the web directly with the users expecting it to be responsive to their needs.[1]

In this project, three modules are created which is used to monitor various environmental parameters and update it real time data to a server. The parameters measured include ambient temperature and humidity of the room, noise levels, the number of people entering and leaving the room and toxic gas detector. In case a flammable gas is detected, an alarm is triggered and an email is sent to the user's account.
\end{abstract}

Arduino is used to integrate and program the hardware components with ESP8266 being the WiFi component which connects to the host webpage. The server side is created on an IoT platform, Ubidots.

\section{General Terms}

Internet of Things(IoT), monitoring, Arduino, ESP8266, Ubidots, wireless sensor networks

\section{Keywords}

Keywords are your own designated keywords which can be used for easy location of the manuscript using any search engines.

\section{INTRODUCTION}

The Internet of Things, Internet of Everything, Machine-toMachine (M2M), Smart Systems etc. all refer to devices or gadgets that are intelligently connected to their users and the web. They consist of an array of data gathering components: embedded sensors, actuators, etc. that generates heaps of useful information to respond to the experience and needs of users. IoT devices are predicted have an impact in all spheres of our lives. As this technology evolves, it is estimated that by the next decade, there will be around 40 billion devices connected to the web. And, when you bring these devices together, organizations can easily collect, analyze and understand device activity and performance via the machine data. [2] The proliferation of these devices creates the Internet of Things (IoT), where sensors and actuators blend seamlessly with the environment, and the information is shared across platforms. [3]

The diversity in the application of the technologies used will result in services that can provide a more efficient model for energy management, environmental factors, or aspects related to the health and welfare of citizens, efficient management of public transport, or just to the monitoring of any parameter of interest, system or service that can be potentially connected to an information network, with the ultimate goal of improving the experience and quality of life for the citizens, the efficient and rational use of the target resource, and at the ensuring a continued and sustainable economic growth [2].Real time environment monitoring systems have a great number of uses and applications. It can be used to monitor storage centres for any gas leakages, extreme changes is temperature or humidity. It can be used to monitor the number of people that visit the room being monitored and extrapolate the data to show the average number of visitors entering the room for the week. Any deviation from the optimum value can trigger an alarm and notify the user to take remedial steps. Such systems are beneficial even at an insurance point of view. Modules can be used to detect hazardous gasses in industrial premises and take appropriate steps to alleviate the problem before it exacerbates.

\section{PRIMARY COMPONENTS USED}

\subsection{Arduino Pro Mini}

In this project, the programmable component used is the Arduino Pro mini 3.3v which is much more compact than a regular Arduino board and can be externally powered by a 9v battery. The Arduino Pro Mini is a microcontroller board based on the ATmega328. It has 14 digital input/output pins (of which 6 can be used as PWM outputs), 6 analog inputs, an on-board resonator, a reset button, and holes for mounting pin headers. A six pin header can be connected to an FTDI cable to provide USB power and communication to the board [4].

\subsection{ESP8266}

The ESP8266 WiFi Module is a self-contained SOC with integrated TCP/IP protocol stack that can give any microcontroller access to a WiFi network. Each ESP8266 module comes pre-programmed with an AT command set firmware, which can be hooked up to the Arduino to get as much WiFi-ability as a WiFi Shield offers. The ESP8266 module is an extremely cost effective board with a huge, and ever growing, community [5].

\section{DESIGN SPECIFICATIONS}

The proposed project is used for real time data analysis of a few physical variables which is divided among three modules. Such a device would be extremely beneficial to keep a check on parameters where constant monitoring is required and any deviation from such would lead to trigger of an alarm and sending of a notification, such as an email, to the user's email account.

\subsection{Module One}

The first module is used for reading temperature and humidity values of the current environment and relaying that data to the Ubidots server, which is an open source online IoT development platform. The average temperature of the day is also computed using this data. The temperature and humidity values are plotted in a graph which updates real time. A Narcoleptic delay library is used to put the pro mini in a low power sleep mode to conserve battery life during the intervals 
when sensing does not take place.The sensor used to retrieve temperate and humidity values is the DHT22, which is a lowcost temperature and humidity sensor. The sensor is given a $5 \mathrm{~V}$ input and its output is connected to an analog pin of the Arduino. The module also has two led laser diodes attached which is used in the counter to estimate the number of people entering the room. These $650 \mathrm{~nm}$ red laser diodes are fitted in copper head and works with a $5 \mathrm{~V}$ supply and a power of $5 \mathrm{~mW}$.

The entire module is powered using a 9V battery. A LM805 voltage regulator is used to supply $5 \mathrm{~V}$ from the battery to power the DHT22 sensor and the laser diodes.

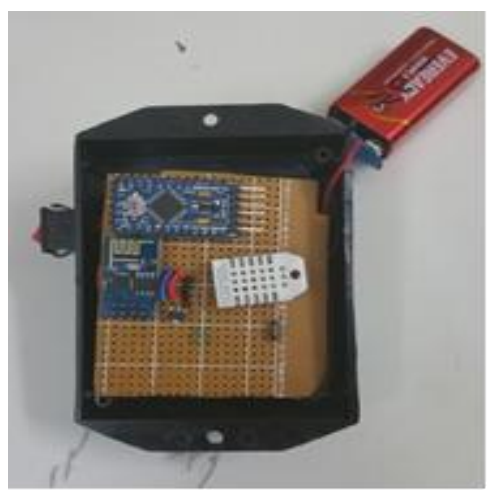

Fig 1: Circuit construction of module one

\subsection{Module Two}

The second module consists of two light dependent resistors which are used for the people counter. Laser light emitting from the laser diodes in the first module falls on these LDRs. A comparison of light intensity is made between the first and second LDR. When the light on the first LDR breaks before the light on the second, the counter increments, signifying a person has entered the room. Similarly, when the light shining on the second LDR first, the counter decrements, signifying that a person has left the room. The number of people currently in the room is displayed on the Ubidots dashboard. Also, the average number of people that entered the room for the week is displayed.

The entire module is soldered and encased in a plastic enclosure. Light falling on the LDR is concentrated by attaching 2 black tubes that reflect the surrounding light onto the LDR. An external switch is provided to switch on and off the module as required.

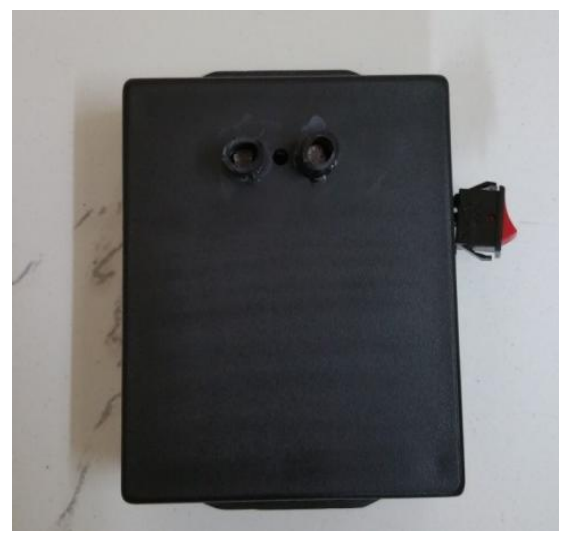

Fig 2: Enclosed view of module two

\subsection{Module Three}

The third module contains sensors to monitor the ambient noise level and the presence of any flammable gas, smoke etc. present in the room. The noise level is observed in a gauge widget present in the Ubidots dashboard and the gas level is plotted in a real time graph. The gas sensor used in the project is the MQ2 sensor. The Gas Sensor (MQ2) module is useful for gas leakage detecting (in home and industry). It can detect H2, LPG, CH4, CO, Alcohol, Smoke, Propane. Based on its fast response time, measurements can be taken as soon as possible [6]. The power supply for the Gas sensor is taken from a $5 \mathrm{~V}$ volt supply drawn from a battery via an LM805 voltage regulator.

The sensor used to monitor the noise levels is the Grove Sound sensor. The main component of the module is a simple microphone, which is based on the LM358 amplifier and an electret microphone. This module's output is analog and can be easily sampled and tested by an Arduino pro mini. A buzzer is attached to a pin of the pro mini which beeps every time the gas level exceeds its optimum values. The ESP8266 is connected to $3.3 \mathrm{~V}$ voltage supply and sends data values periodically to the dashboard.

The module is soldered into a strip board and encased into a plastic case designed according to the measurement of the board. The module is connected to $4 \mathrm{AA}$ batteries which supplies sufficient stable current to power the module. The module can be switch on and off as required. A buzzer is attached to the module externally.

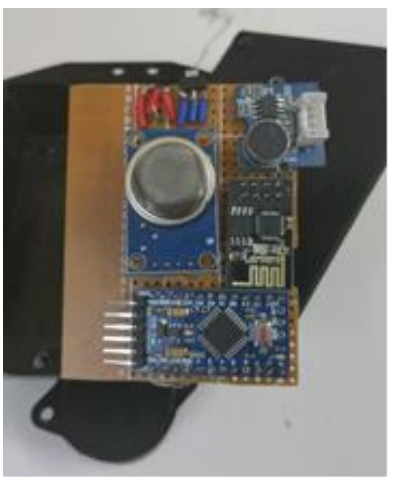

Fig 3: Circuit construction of module three

\section{UBIDOTS}

Ubidots is a platform for connecting people, processes and things. It allows companies and cities to become more efficient by capitalizing on information from interconnected assets and processes. Ubidots is a cloud service to store and analyse sensor data in real-time, it is free with 30,000 dots/month data-points usage, features as follow: push data from internet enabled device, display sensor data through widgets, data is updated in real time and no need to refresh the browser, trigger alerts when a sensor data hits a value and it has a powerful and flexible API [7].

A dashboard was created on the Ubidots website to provide widgets for monitoring and plotting real time graphs and data of the sensors which are being implemented in the project. It was also used to trigger alarm when a variable exceeds its optimum value. 

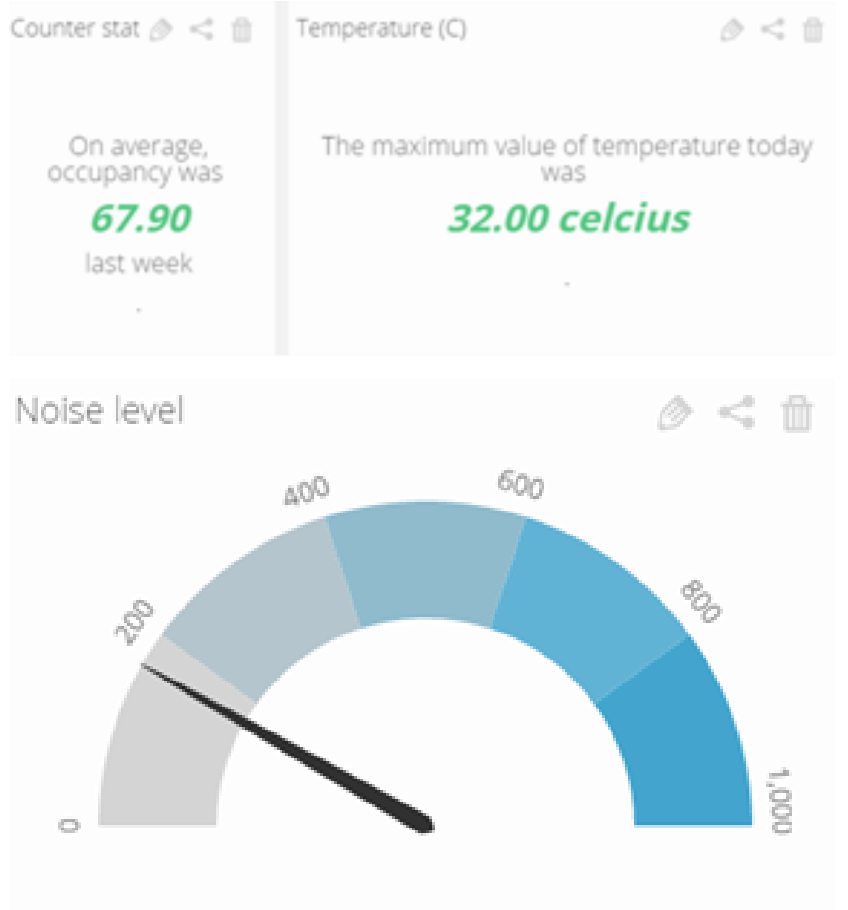

Fig 4: Widgets on Ubidots dashboard

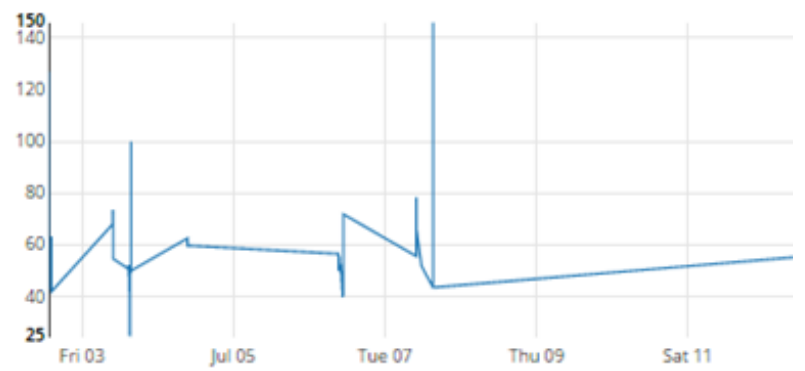

Fig 5: View of data points for a source variable
5. CONCLUSION AND FUTURE SCOPE

The application of the project is numerous and can be further developed to integrate more sensors which are applicable to its use in environment monitoring. It can also be used to trigger an external event or control a remote device which can be even used to mitigate critical situations. Such an external event can be such as turning the sprinkler on when there is fire or smoke detected, turning a fan on in a room when it gets too hot etc. The project can further be reduced to much smaller sizes and integrated in just a single chip which can be in the form of miniature tags.

\section{REFERENCES}

[1] Techopedia.com, 'What is the Internet of Things (IoT)? Definition from Techopedia', 2015. [Online]. Available: https://www.techopedia.com/definition/28247/internetof-things-iot.

[2] C. Riley, 'Machine Data for End-to-End IoT System Monitoring', Blog.logentries.com, 2015. [Online]. Available: https://blog.logentries.com/2015/02/machinedata-for-end-to-end-iot-system-monitoring/.

[3] J. Gubbi, R. Buyya, S. Marusic and M. Palaniswami, 'Internet of Things (IoT): A vision, architectural elements, and future directions', Future Generation Computer Systems, vol. 29, no. 7, pp. 1645-1660, 2013.

[4] Arduino.cc, 'Arduino - ArduinoBoardProMini', 2015. [Online].Available:https://www.arduino.cc/en/Main/Ard uinoBoardProMini.

[5] Sparkfun.com, 'WiFi Module - ESP8266 - WRL-13678 SparkFun Electronics', 2015. [Online]. Available: https://www.sparkfun.com/products/13678.

[6] Seeedstudio.com, 'Grove - Gas Sensor(MQ2) - Wiki', 2015.[Online].Available:http://www.seeedstudio.com/wi ki/Grove_-_Gas_Sensor(MQ2).

[7] Pulsobase.com, 'Ubidots | Pulso Base', 2015. [Online]. Available: http://pulsobase.com/core/?listing=ubidots. 